\title{
Research on Digital Watermark Algorithm based on Compression Perception
}

\author{
Junkai Zhang \\ (Software Technology Vocational College, North China University of Water \\ Resources and Electric Power, Zhengzhou 450045, China) \\ henanzjk@126.com
}

\begin{abstract}
How to improve the effect of digital watermark has been the main direction of research. This paper first proposes a network adaptive sparse transformation method based on dictionary learning. In this method, the adaptive optimal sparse transform is constructed, and the sparse matrix of the network data is introduced into the dictionary learning problem to satisfy the requirement of the data processing of the watermark image and the high real time requirement of the sparse transform. Through the improvement of the watermark embedding and extraction, the compression perception algorithm has achieved good results in robustness and concealment. The simulation results show that the algorithm has good feasibility.
\end{abstract}

Keywords: compression perception; digital watermark; dictionary learning

\section{Introduction}

Digital watermark technology is a branch of information security, which has a very important role in image even copyright. Digital watermark technology is to embed the watermark information into some kinds of media data, so as to provide security guarantee for the media data [1-2]. Scholars in China and abroad have researched the digital watermark in different degrees. In reference [3], a semi fragile digital watermark algorithm for color image restoration is proposed, and the algorithm can accurately detect the tampered image, locate and restore. Simulation results show that the proposed algorithm cannot only be used for authentication and recovery of color images, but also has a good vulnerability to conventional signal processing operations. The reference [4] proposes a digital watermark algorithm based on discrete wavelet transform which has been improved even. The original algorithm has scrambled the Arnold of the watermark, while the algorithm in this paper uses a better security chaos scrambling. The original algorithm strength embeds 2 kinds of watermark in the high and low frequency parts, respectively, while the new algorithm is used in this way in order to improve the invisibility and robustness of the watermark; The reference [5] proposes HVS-Curvelet algorithm, and experiments show that the HVS-Curvelet algorithm can really promote the digital watermark algorithm or not. The reference [6] proposes an improved digital watermark technique which based on wavelet transform. First, discrete two-dimensional wavelet decomposition and reconstruction algorithm is used to decompose and decompose the image. Second, image watermark is loaded, embedded and extracted by image scrambling algorithm and low frequency coefficients in the wavelet domain. Experimental results show that the proposed algorithm has good robust performance, and effectively ensures the safety of the image; A digital image watermark algorithm based on discrete chaotic dynamical system and wavelet 
decomposition is designed in the reference [7]. In the watermark image pre processing stage, the chaotic sequences generated by Logistic map are used to extend scrambling the watermark image, and then use the Logistic map to generate the key to encrypt the watermark information, so as to ensure the security of the watermark information. In the stage of watermark embedding, the three layer wavelet decomposition of the host image is decomposed, and the watermark is embedded into the high frequency coefficient of the horizontal direction by the wavelet coefficient. The reference [8] proposes a multi sensor image fusion and dual watermark algorithm based on wavelet packet domain, and the experimental results show that the fusion image can effectively improve the spatial resolution which based on the spectral information of multispectral images; the fusion image with the watermark is not visible and robust.

This paper proposes a digital watermark technology in compression perception. Through the adaptive optimal sparse transform constructed, the sparse matrix of the network data is introduced into the dictionary learning problem to satisfy the requirement of the data processing of the watermark image and the high real time requirement of the sparse transform. In the watermark algorithm, noise attacks, filtering attacks and compression attacks have a good performance.

\section{Digital Watermark}

Digital watermark technology is a kind of technology which is embedded in the digital media by means of some identification information, while the identification information is called digital watermark. Today, especially in cloud computing, digital watermark and digital signature technology are the same as belong to an important part of the information hiding, and they can play a role in protecting the security of information, so digital watermark technology is particularly important. Digital watermark contains three basic elements: the structure of the watermark itself, the watermark embedding algorithm and watermark detection or extraction algorithm. There is no uniform standard for the watermark itself, which is related to the requirement of the environment. Watermark embedding algorithm such as $I_{w}=E(I, W)$, in the algorithm $E$ is watermark embedding function, $I$ is original image, $W$ is watermark information, $\widehat{W}$ is processed watermark, $\hat{W}=F(I, W, K), K$ is cipher code, watermark extraction function is $D, W^{*}=D\left(I_{w}, I, K\right), W^{*}$ is extracted watermark. Digital watermark embedding and extraction as shown in Figure 1-2.

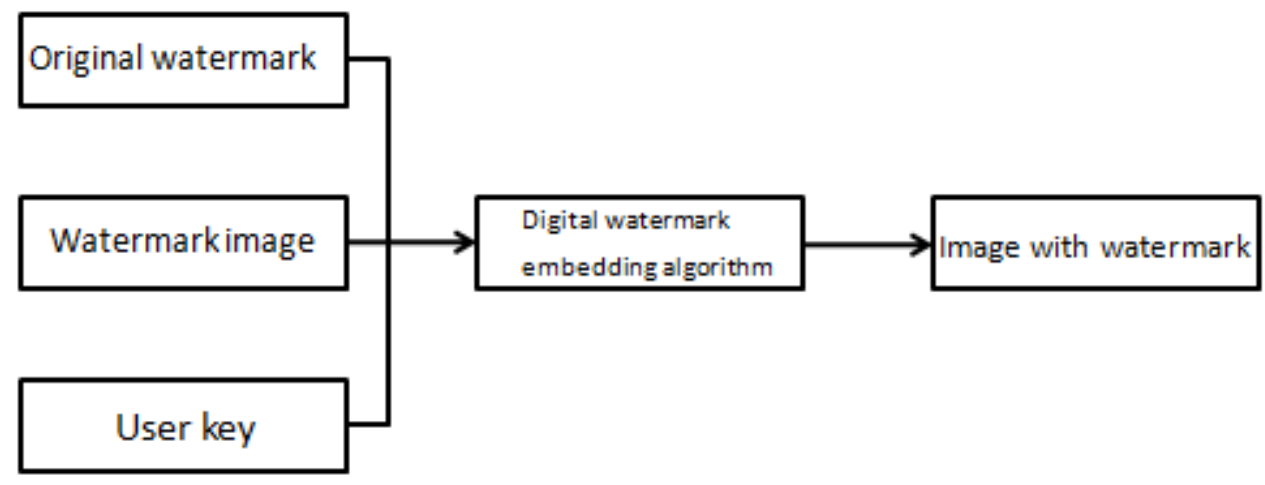

Figure 1. Embedding of Digital Watermarking 


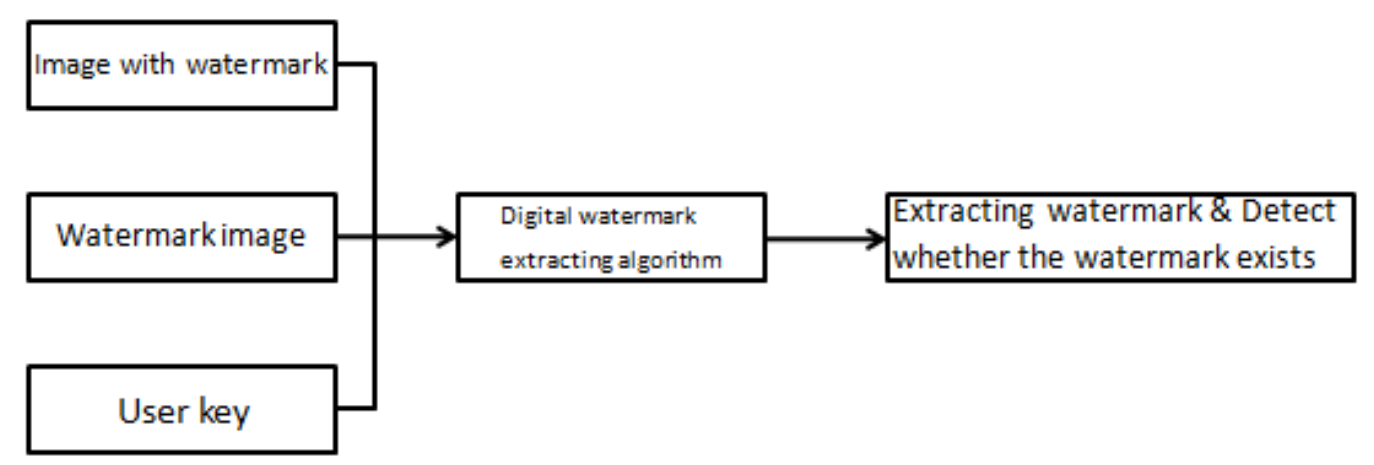

Figure 2. Extraction of Digital Watermarking

\section{Adaptive Compression Perception Theory}

\subsection{Compression Perception}

Compression perception is the theory to combine sampling and compression. Sample shall be done first and then compress storage in the process of signal processing. The process is bound to result in a large number of data lost and serious waste of resources. Especially for the image which is relatively high in the cloud computing, the traditional compression theory certainly causes a lot of redundant data, which will undoubtedly increase the cost of network transmission.

Suppose the number of nodes in the network is $W$, node for $m$ sampling, the data length of each node is $m$. Each node is represented by a matrix representation, which is shown in Formula (1)

$$
X_{D}=\left(\begin{array}{ccc}
x_{11} & \cdots & x_{1 W} \\
\vdots & & \vdots \\
x_{m 1} & \cdots & x_{m W}
\end{array}\right)
$$

The node data in the Formula (1) is written in vector form as follows:

$$
\begin{aligned}
X & =V\left(X_{D}\right) \\
& =\left(x_{11}, \ldots, x_{1 N}, x_{12}, \ldots, x_{n 2}, x_{1 m}, \ldots, x_{m W}\right)^{T} \\
& =(x(1), x(2), \ldots x(K))^{T}
\end{aligned}
$$

(2)

In which $X \in R^{K}, K=m \times W$.

As the data collected by nodes in the network is not sparse, the data of the network nodes are sparse representation before the compression perception is carried out. The $\boldsymbol{\Psi}$ is the dimensional sparse matrix with the length of $N$, vector $\boldsymbol{\theta}$ contains 
$K$ nonzero elements, and then the sparse results are as follows:

$\boldsymbol{X}=\boldsymbol{\Psi} \boldsymbol{\theta}=\sum_{i=1}^{N} \psi_{i} \theta_{i}=\sum_{k=1}^{K} \psi_{k} \theta_{k}$

(3)

The $m \times n$ measurement matrix generated by the node $w(w \in\{1,2, \cdots W\})$ is set to $\gamma_{w}$. The measurement value of each node is written as vector form:

$Y=\left(y_{11}, \ldots, x_{1 m}, y_{12}, \ldots, y_{m 2}, y_{1 w}, \ldots, y_{m W}\right)^{T}=(y(1), y(2), \ldots y(M))$

(4)

The network data measurement process can be expressed by the following matrix vector:

$Y=\gamma \Psi \theta$

(5)

In which, $\boldsymbol{Y} \in R^{M}, M=J \times w$, the measurement matrix is as follows:

$\Phi=\left(\begin{array}{cccc}\Phi_{1} & 0 & \cdots & 0 \\ 0 & \Phi_{2} & \cdots & 0 \\ \vdots & \vdots & \ddots & \vdots \\ 0 & 0 & \cdots & \Phi_{w}\end{array}\right)$

(6)

In order to recover the data, the data of these compressed data needs to be reconstructed, and the accuracy of the node data reconstruction is directly determined by the sparsity of the sparse vector $\boldsymbol{\theta}$ of the network data.

\subsection{Data Sparse based on Dictionary Learning}

Set training sample value is $\left\{x_{1}, x_{2}, \cdots x_{i}\right\}$, transform the sparse representation of dictionary learning into the problem of solving the objective function with the dictionary vector as the variable, within, $X_{T i}$ is the training value matrix which is composed of training individuals $x_{i}, \boldsymbol{\Psi}$ is sparse dictionary, $\boldsymbol{\Theta}=\left\{\theta_{i, j}\right\}$ is sparse matrix representation, Sparse metric is $J_{\rho}(\boldsymbol{\Theta})$, therefore, it is needed to find the minimum value of the objective function, such as formula (6)

$\arg \min _{\boldsymbol{\theta}}\left(\left\|X_{T i}-\boldsymbol{\Psi} \Theta\right\|^{2}\right)+\lambda J_{\rho}(\boldsymbol{\Theta})$

In order to better express the data of the dictionary learning sparse understanding, 
this section needs to define the signal compressibility. Set signal element $s$ to meet

$$
|s|_{\zeta} \leq \tau k^{-r}
$$

In which, $|s|_{\zeta}$ is $\zeta$ element of signal $s . r \geq 1, \tau$ is constant. As a result, if the matrix $S$ element which meets the requirements of Formula (7), matrix can be compressed. $S_{\zeta}$ shows that only $\zeta$ elements with the largest amplitude are stored in matrix $S . S_{\zeta}$ is the optimal approximation of matrix $\zeta$. The approximate errors are as follows:

$\left\|S-S_{\zeta}\right\|_{F} \leq \tau K^{-r}$

(9)

$$
\boldsymbol{\Psi}=S \boldsymbol{V}
$$

Through the definition of above, dictionary $\boldsymbol{\Psi}$ can be expressed as full rank matrix $\boldsymbol{V}$ by $d \times M$. dictionary $\boldsymbol{\Psi}$ can be called compression dictionary.

Combined with the data of (5) and (9), sparsity structure can be converted into the following problem: through the given training sample matrix $X_{T i}$ and full rank matrix, estimate generated matrix $\mathbf{S}$, namely by solving the minimum value of the objective function $v(\mathbf{\Theta}, \mathbf{S})$ with the independent variables of $(\boldsymbol{\Theta}, \mathbf{S})$ to get the sparse matrix.

$$
\arg \min _{S}\left\{\min _{\Theta} v(\boldsymbol{\Theta}, \boldsymbol{S})\right\} \text { : }
$$

$$
v(\boldsymbol{\Theta}, \boldsymbol{S})=\left\|\boldsymbol{V S} \boldsymbol{\Theta}-\boldsymbol{X}_{\boldsymbol{T R}}\right\|^{2}+\lambda J_{p}(\boldsymbol{\Theta})+\gamma J_{q}(\boldsymbol{S})
$$

In which $\rho \in\{p, q\} \leq 1 . \lambda$ is the weights of transform coefficients. $\gamma$ is the weights of the sparse degree of generated matrix, $p=q=1$.

\subsection{Algorithm Description}

In order to solve the minimum value of Formula (10), this paper uses $\left(\boldsymbol{\Theta}^{[0]}, \mathbf{S}^{[0]}\right)$ to show the iterative initial value. The algorithm makes the objective function value decreases through optimizing the solution of $\boldsymbol{\Theta}^{[n]} \rightarrow \boldsymbol{\Theta}^{[n+1]}$ or $\boldsymbol{S}^{[n]} \rightarrow \boldsymbol{S}^{[n+1]}$. So set variables and update equation.

When variable $\mathbf{S}$ is fixed, record the objective function as $v_{\mathbf{S}}(\boldsymbol{\Theta})$ and the majorizing function can be recorded as

$$
\mu_{\mathbf{S}}\left(\boldsymbol{\Theta}, \boldsymbol{\Theta}^{[n]}\right)=\operatorname{tr}\left\{c_{\mathbf{V}} c_{\mathbf{S}} \boldsymbol{\Theta}^{T} \boldsymbol{\Theta}-2 \boldsymbol{\Theta}^{T}\left(\mathbf{S}^{T} \mathbf{V}^{T}\left(X_{T i}-\lambda V S \boldsymbol{\Theta}^{[n]}\right)\right)\right\}
$$


Note that $\mu_{\mathrm{S}}$ is nonsmooth convex function, so the matrix in the direction of the minimum gradient of $\mu_{\mathrm{s}}$, that

$0 \in \partial \mu_{S}\left(\boldsymbol{\Theta}^{[n+1]}, \boldsymbol{\Theta}^{[n]}\right)$

It can be got by Formula (11)

$$
\partial \mu_{\mathbf{S}}\left(\boldsymbol{\Theta}, \boldsymbol{\Theta}^{[n]}\right)=c_{\mathbf{V}} c_{\mathbf{S}} \boldsymbol{\Theta}-\left(\mathbf{S}^{T} \mathbf{V}^{T} X_{T i}-\lambda V S \boldsymbol{\Theta}^{[n]}+\lambda c_{\mathbf{V}} c_{\mathbf{S}} \boldsymbol{\Theta}^{[n]}\right)
$$

Put Formula (13) in to

$\boldsymbol{\Theta}^{[n+1]}=S_{\lambda / 2}\left[\frac{1}{c_{\boldsymbol{V}} c_{S}}\left(\boldsymbol{S}^{T} \boldsymbol{V}^{T}\left(\boldsymbol{X}_{\boldsymbol{T R}}-\boldsymbol{V} \boldsymbol{S} \boldsymbol{\Theta}^{[n]}\right)+c_{V} c_{\boldsymbol{S}} \boldsymbol{\Theta}^{[n]}\right)\right]$

In which, $S_{\alpha}$ is contraction factor, for matrix $\mathbf{A}=\left\{a_{i, j}\right\}$ :

$S_{\alpha}(\mathbf{A})=\left\{\begin{array}{cc}a_{i, j}-\operatorname{sign}\left(a_{i, j}\right) \cdot \alpha / 2 & \alpha / 2<\left|a_{i, j}\right| \\ 0 & \alpha / 2>\left|a_{i, j}\right|\end{array}\right.$

Similarly, when variable $\boldsymbol{\Theta}$ is fixed, variable $\mathbf{S}$ updates equation as follows:

$$
\boldsymbol{\Theta}^{[n+1]}=S_{\lambda}\left[\frac{\mathbf{S}^{T} \mathbf{V}^{T}\left(\left(X_{T i}-\lambda V S \boldsymbol{\Theta}^{[n]}\right)\right)}{c_{\mathbf{V}} c_{\mathbf{S}}}++c_{\mathbf{V}} c_{\mathbf{S}} \boldsymbol{\Theta}^{[n]}\right]
$$

In which, $S_{\alpha}$ is given by (16).

\section{Digital Watermark Algorithm based on Compression Perception}

This paper realizes the digital watermark embedding and extraction in compression perception, uses dictionary learning for watermark embedding, which can guarantee the algorithm has good robustness. The matrix is used as the key. The watermark extraction 
does no longer need the original image, which can save a lot of storage space and has obvious advantages.

\subsection{Watermark Embedding Algorithm}

The watermark with different intensity can embedding in different parts of the image. The watermark strength will affect the robustness and the concealment of the watermark which are a pair of contradictory characteristics. To achieve the balance, one of the characteristics shall be satisfied as much as possible under the premise of the other one is guaranteed. Because each image is not uniform. There is rich texture in some parts and plain texture of some other places of the image. So the image is divided into several blocks. The several decomposition pieces are of poor uniformity, some parts are in good uniformity while some parts are in poor uniformity. The parts with poor uniformity contain relatively good energy which are suitable for greater watermark embedding and can ensure the robustness of watermark. The original image $I(m \times m)$ is divided into blocks and get the non uniformity value for each block according to Formula (17).

$$
d\left(B_{k}\right)=\frac{\sum_{(i, j) \in B_{k}} \frac{\left|f(i, j)-m_{k}\right|}{m_{k}^{1+\tau}}}{b^{2}}
$$

In the formula, $B_{k}$ is the sub-block of $n \times n, m_{k}$ is the average of $B_{k}, \tau$ is correction factor. The uniformity of the sub-block is different along with the increase of $d\left(B_{k}\right)$. L sub-blocks with large amount are selected to embedding in lager watermark $L=(1,2 \ldots(m \times m) /(n \times n))$. Sparse the $L$ sub-blocks by compression perception and dictionary learning. Change matrix as the perception matrix to get the measurement value $y_{i}$. As a result, there are $L$ sub-blocks and $L$ measurement values. Perception matrix $\Phi_{L}$ is stored as the key. Since the size of the watermark is $n \times n$, according to the theory of image quantitative, Formula (19) is used to modify the measurement values.

$$
\hat{y}_{i}=\left\{\begin{array}{l}
\left(\lambda_{1}-\frac{1}{2}\right) \delta, \text { if }\left(\lambda_{i}+w_{n}\right) \% 2=1 \\
\left(\lambda_{1}+\frac{1}{2}\right) \delta, \text { if }\left(\lambda_{i}+w_{n}\right) \% 2=0
\end{array}\right.
$$

In the formula, $y_{i}$ is the measurement value of the watermark which is to be embedded. $\hat{y}_{i}$ is the measurement value of the watermark which is embedded. $\lambda_{i}=\left\lfloor\frac{y_{i}}{\delta}\right\rfloor$. It can be found from the formula that the quantization step length is mainly decided by $d\left(B_{k}\right)$. The greater its value is, the larger the quantization step length is, the more watermark can be embedded. This formula has good security, robustness and 
concealment.

\subsection{Watermark Extraction}

The extraction and embedding of the watermark is a mutual converse process. Decompose an image with watermark into a certain size of modules. According to Formula (17), calculate the non uniformity rate of each sub-block and select $L$ image blocks. With the help of the key when the watermark embedding, select $L$ sub-blocks. The expression is as follows:

$\hat{w}_{n}=\left\{\begin{array}{l}1, i f(\hat{\lambda} \% 2=1) \\ 0, f(\hat{\lambda} \% 2==0)\end{array}\right.$

In the formula, $\hat{\lambda}=\left\lceil\frac{y_{i}}{\delta}\right\rceil$, the watermark extraction algorithm is as follows:

$$
\begin{aligned}
& \text { Input }: \hat{y}_{i}, \hat{d}\left(B_{k}\right) ; \\
& \text { Output }: \hat{w}_{n} ; \\
& \text { for } i=1 \text { to } L \times(256 \times \sigma) d o \\
& \delta=a \hat{d}\left(B_{k}\right) \rightarrow \delta ; \\
& \hat{\lambda}_{i}=\text { floor }\left(\hat{y}_{i} / \delta\right) \rightarrow \hat{\lambda}_{i} \\
& \text { if }\left(\hat{\lambda}_{i} \% 2\right)=1 \text { then } \hat{w}_{n}=1 \\
& \text { else } \hat{w}_{n}=0 ; \\
& \text { get } \hat{w}_{n} ; \\
& \text { else } \hat{w}_{n}=0 ; \\
& \text { get } \hat{w}_{n} ; \\
& i++; \\
& \text { end for }
\end{aligned}
$$

\section{The Experimental Simulation}

In order to better verify the effectiveness of the algorithm, the hardware configuration is Intel core $\mathrm{i} 3$, hard disk is $320 \mathrm{G}$ and the memory is 4GDDR3. Visual c + + and Matlab programming are selected as the software environment.In order to better demonstrate the algorithm in this paper has very good concealment to the image, the method in this paper which is changeable domain algorithm is compared with spatial domain algorithm. This paper also selects SSIM [9] as the evaluation standard of the static image quality, uses $I$ to show the original image and $I$ as the image with watermark. The comparing results are shown in Figure 3. 
$S(I, \hat{I})=f(l(I, \hat{I}), c(I, \hat{I}), d(I, \hat{I}))$

In the formula, $l(I, \hat{I})$ is the brightness contrast function with the rate of $l(I, \hat{I})=\frac{2 l(I) l(\hat{I})+c_{1}}{l(I)^{2}+l(\hat{I})^{2}+c_{1}} \cdot l(I)$ is the average value of the original image and $l(\hat{I})$ is the average value of the image with watermark. $c(I, \hat{I})$ is the contrast function with rate of $c(I, \hat{I})=\frac{2 c(I) c(\hat{I})+c_{2}}{c(I)^{2}+c(\hat{I})^{2}+c_{2}} \cdot c(I)$ is the variance of the original image and $c(\hat{I})$ is the variance of the image with watermark. $d(I, \hat{I})$ is structure function. $d(I, \hat{I})=\frac{2 d(I) d(\hat{I})+c_{3}}{d(I)^{2}+d(\hat{I})^{2}+c_{3}} \cdot d(I)$ is the covariance of the original image and $d(\hat{I})$ is the covariance of the image with watermark.

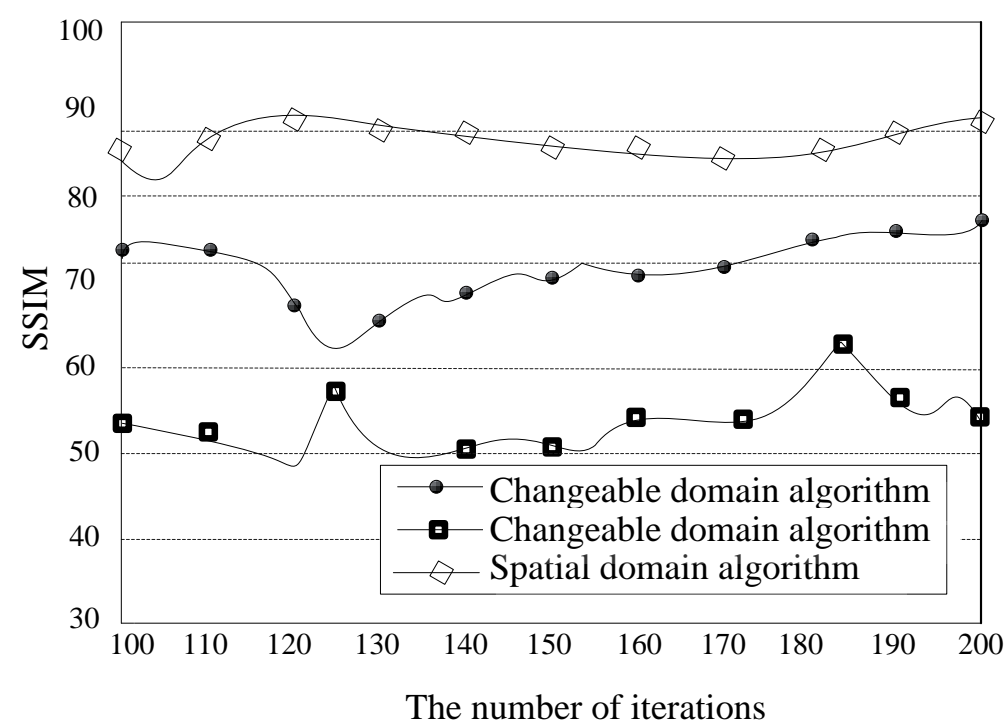

Figure 3. Comparison Effects of Three Algorithms

\section{Conclusion}

This paper researches the watermark algorithm of compression perception and introduces dictionary learning into compression perception algorithm. The improved algorithm is used in watermark embedding and watermark extraction. Through 
simulation experiment, in the comparison of two aspects of robustness and concealment, this paper explains that the algorithm has good feasibility.

\section{References}

[1] Candes E. J. and Wakin M. B., "An introduction to compressive sampling [J]", IEEE Signal Processing Magazine, vol. 25, no. 2, (2008), pp. 21-30.

[2] Huang H. C., Chang F. C., Wu C. H. and Lai W. H., "Watermarking for compressivesampling applications[C]", Proceedings of the 2012 8th International Conference on Intelligent Information on Hiding and Multimedia SignalProcessing, Washington:IEEE Press, (2012), pp. 223-226.

[3] Tan chun-jiao and Zhu en, "An auto-restorable semi-fragile watermarking algorithm for color image[J]", Computer Engineering and Science, vol. 37, no. 3, (2015), pp. 594-59.

[4] Zhang qin and Cui li, "Digital watermarking algorithm based on DWT[J]", Journal of Beijing Normal University (Natural Science), vol. 51, no. 1, (2015), pp. 19-22.

[5] Li jia, "Digital Watermarking Algorithm Research Based on HVS-Curvelet[J]", .Bulletin of Science and Technology, vol. 30, no. 3, (2014), pp. 211-214.

[6] L. liang and Chen chang-zhi, "An Improved Wavelet Transforms Image Digital Watermarking Technology[J]”, Bulletin of Science and Technology, vol. 28, (2012), pp. 144-148.

[7] Chen he-shan, lv zhen-zhen, Luo wei, "Digital Image Watermarking Algorithm Based on Dispersed Chaotic Mapping System[J]", Computer Science, (2014), vol. 41, no. 12, pp. 48-52.

[8] Li chen-e and Ban hao, "Method of multisensor image fusion and digital watermarking[J]", Chinses Journal of Liquid Crystals and Displays, vol. 29, no. 2, (2014), pp. 286-291.

[9] Tong yu-bin, zhang qi-shan and Qi yun-pin, "Image Quality Assessing by Combining PSNR with SSIM[J]”, JOURNAL OF IMAGE AND GRAPHICS, vol. 11, no. 12, (2006), pp. 1758-1763.

\section{Author}

Junkai Zhang (1982.4-) Assistant, Master, Research Orientation: Computing Network; 